\title{
Carrier Lifetime and Mobility Enhancement in Nearly Defect-Free Core-Shell Nanowires Measured Using Time-Resolved Terahertz Spectroscopy
}

\author{
Patrick Parkinson, ${ }^{\dagger}$ Hannah J. Joyce, ${ }^{\ddagger}$ Qiang Gao, ${ }^{\ddagger}$ Hark Hoe Tan, ${ }^{\ddagger}$ Xin Zhang, ${ }^{\S}$ \\ Jin Zou, ${ }^{\S}$ Chennupati Jagadish, ${ }^{\ddagger}$ Laura M. Herz, ${ }^{\dagger}$ and Michael B. Johnston ${ }^{\star, \dagger}$ \\ Clarendon Laboratory, Department of Physics, University of Oxford, Parks Road, \\ Oxford, OX1 3PU, United Kingdom, Department of Electronic Materials Engineering, \\ Research School of Physics and Engineering, Institute of Advanced Studies, Australian \\ National University, Canberra ACT 0200, Australia, and School of Engineering and \\ Centre for Microscopy and Microanalysis, The University of Queensland, St Lucia, \\ QLD 4072, Australia
}

Received May 23, 2009; Revised Manuscript Received July 9, 2009

\begin{abstract}
We have used transient terahertz photoconductivity measurements to assess the efficacy of two-temperature growth and core-shell encapsulation techniques on the electronic properties of GaAs nanowires. We demonstrate that two-temperature growth of the GaAs core leads to an almost doubling in charge-carrier mobility and a tripling of carrier lifetime. In addition, overcoating the GaAs core with a larger-bandgap material is shown to reduce the density of surface traps by $82 \%$, thereby enhancing the charge conductivity.
\end{abstract}

Semiconductor nanowires are promising new materials for implementation in nanoscale electronic and optoelectronic devices. Of particular interest are III-V semiconductor nanowires, which can exhibit a direct bandgap and a high electron mobility. ${ }^{1}$ However, the large surface-to-volume ratio inherent to nanowires results in the presence of surface traps offering easy access to carrier and exciton recombination pathways. ${ }^{2,3}$ In addition, one-temperature growth techniques have been shown to cause a significant twin-defect (stacking-defect) density within the nanowires. ${ }^{4}$ Refinements in the epitaxial growth of these nanowires are therefore essential in order for their optoelectronic and crystallographic standards to approach those of bulk material. ${ }^{2,6,7}$ Such efforts are complicated by the fact that electrical measurements conducted on nanowires to determine charge-carrier mobility are often obscured by properties of the electrical contacts. Most contactless spectroscopic probes of nanowires to date have relied upon low-temperature photoluminescence measurements to characterize optoelectronic quality by measuring excitonic dynamics and radiative quantum efficiency., ${ }^{2,6}$ However, for use of these materials in nanoelectronics and

\footnotetext{
* To whom correspondence should be addressed. E-mail: m.johnston@ physics.ox.ac.uk.

${ }^{\dagger}$ University of Oxford.

* Australian National University.

$\S$ The University of Queensland.
}

optoelectronics, it is essential to determine charge-carrier mobility and lifetime at room temperature.

In this study, we have conducted transient photoconductivity measurements on an ensemble of nanowires in order to assess the effect of nearly defect-free (two-temperature) growth and core-shell encapsulation technologies on charge-carrier trapping and mobility. Optical-pump terahertz-probe spectroscopy was employed as a noncontact ultrafast probe of the room-temperature photoconductivity with subpicosecond resolution. We demonstrate that both two-temperature growth and encapsulation of the GaAs nanowires with a higher band gap material lead to significant increases in the lifetime of free charge carriers. Encapsulation of the nanowires is shown to be highly effective, reducing the areal density of surface traps to one-seventh of that for the untreated wires. Importantly, we find that moving from one-temperature growth to a two-temperature procedure (comprising a brief high-temperature step for nucleation and a longer lower-temperature phase for prolonged growth ${ }^{4}$ ) increases the intrinsic carrier mobility of the wires from 1200 $\mathrm{cm}^{2} /(\mathrm{V} \mathrm{s})$ to $2250 \mathrm{~cm}^{2} /(\mathrm{V} \mathrm{s})$.

All nanowire samples were initially grown onto a GaAs substrate as shown in a representative scanning electron microscopy (SEM) image in Figure 1. A gold-colloid seeded vapor-liquid-solid metal-organic chemical vapor deposition (MOCVD) technique was used, as described in refs 2, 4, 6, 


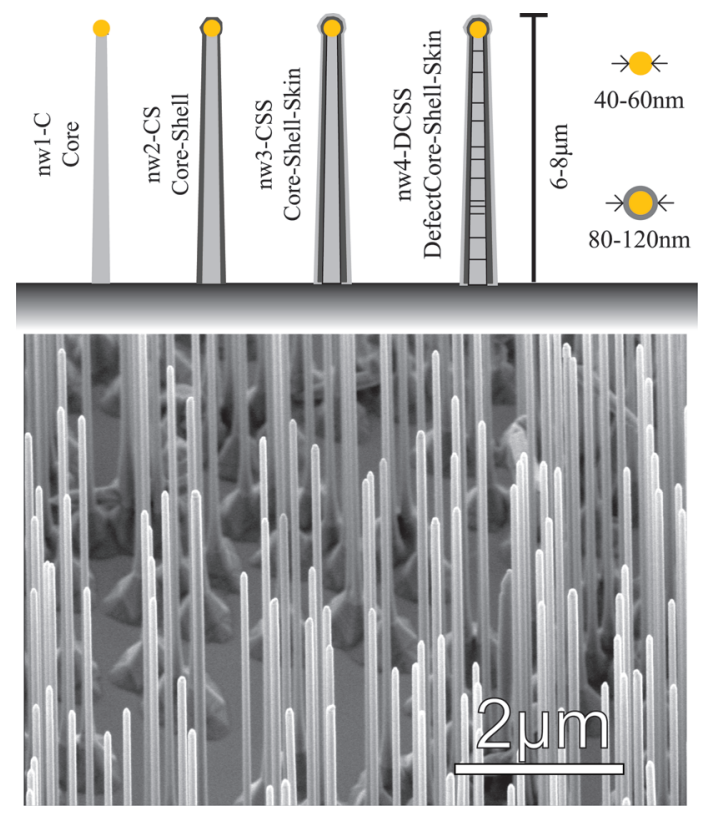

Figure 1. (Above) A schematic representation of the nanowire structures examined in this study along with their typical dimensions. (Below) An SEM image of as-grown [nw3-CSS] nanowires. The scale bar represents $2 \mu \mathrm{m}$, and the image was taken at $40^{\circ}$.

and 8. To eliminate the otherwise dominant photoconductivity of the GaAs substrate, the nanowires were then transferred from the as-grown substrate to a z-cut quartz substrate by gently rubbing the two substrates together. In order to investigate the effects of growth conditions and encapsulation, four different types of nanowire structure were selected, as shown in Figure 1. The first three types were fabricated by the two-temperature growth technique ${ }^{4}$ and subjected to different overcoating procedures: sample [nw1-C] comprises an ensemble of GaAs nanowires of core diameter 40-60 $\mathrm{nm}$ and of 6-8 $\mu \mathrm{m}$ in length; sample [nw2-CS] had an additional AlGaAs shell of thickness $\sim 30 \mathrm{~nm}$ added to encapsulate the wires, while sample [nw3-CSS] had a further GaAs "skin" layer of approximately $5 \mathrm{~nm}$ thickness deposited on the nanowires. AlGaAs shells were grown with a gas phase $\mathrm{Al}$ composition of $26 \% .{ }^{4}$ Measurements of the lowtemperature exciton lifetime in similar structures have shown that the skin layer reduces the oxidation of the AlGaAs shell, thereby reducing oxygen absorption into the GaAs core. ${ }^{2}$ A fourth sample, [nw4-DCSS], was grown by a single-step onetemperature procedure associated with high twin-defect density, and overcoated with an AlGaAs shell layer and a GaAs skin layer in the same way as sample [nw3-CSS]. Thus comparison of the transient conductivity for the first three samples allows conclusions to be drawn on the efficacy of surface treatments, while differences between samples [nw3CSS] and [nw4-DCSS] allow a comparison to be made between the two growth techniques for the core.

The TEM images of Figure 2 show that nanowires grown by the one- and two-temperature procedures have different morphologies. Those grown by the one-temperature procedure are tapered, with thick bases and rough faceted sidewalls, as illustrated in Figure 2c. Those grown by the two-temperature procedure are minimally tapered, with

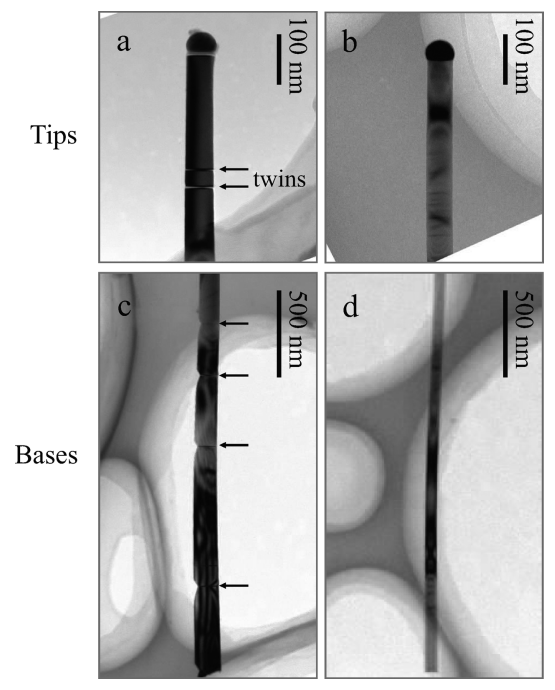

Figure 2. TEM images showing the tips and bases of nanowires grown by $(\mathrm{a}, \mathrm{c})$ the single temperature procedure and $(\mathrm{b}, \mathrm{d})$ the twotemperature procedure. The single temperature procedure results in numerous twin defects, as indicated by arrows in $(\mathrm{a}, \mathrm{c})$. The twotemperature procedure, in comparison, produces the twin-free nanowires of $(b, d)$. Nanowires grown by the single-temperature procedure are considerably more tapered, as evidenced by the wide base diameter in (c), and have rough, faceted sidewalls. Nanowires grown by the two-temperature procedure, in contrast, have smooth sidewalls and are minimally tapered, as evidenced by the narrow base diameter in (d).

smooth sidewalls, as illustrated in Figure 2d. If shell growth is performed at low temperatures, such variations in nanowire morphology may affect shell growth; therefore to achieve uniform, conformal deposition of AlGaAs shells, we use a high growth temperature of $650{ }^{\circ} \mathrm{C} .5$ Thus, the compositional uniformity of the AlGaAs shell should not be affected by these variations in nanowire core morphology. For all core-shell samples, regardless of whether grown by the oneor two-temperature procedure, the shell thickness is approximately $30 \mathrm{~nm}$, and the shell surface is smooth.

To measure the photoconductivity of GaAs nanowires on a picosecond time scale, an optical-pump terahertz-probe spectroscopy system was employed (see Supporting Information for a detailed description of the experimental apparatus). Sample excitation was carried out with a pump pulse of center wavelength $810 \mathrm{~nm}$ and duration $\sim 50 \mathrm{fs}$. The pumpinduced change in transmission of a weak terahertz probe was measured as a function of pump-probe delay, which for the comparable case of a uniformly excited thin film is proportional to the photoinduced conductivity. ${ }^{10}$ For GaAs nanowires, we have shown previously that the carrier scattering rate (and therefore mobility) stabilizes over the first $300 \mathrm{fs}$ after photoexcitation. ${ }^{3}$ We therefore assume in the following analysis that at times $>300$ fs the mobility is constant and any change in photoconductivity is caused solely by a variation in carrier density. ${ }^{3}$

Figure 3 demonstrates that the photoconductivity decay is strongly dependent upon the growth conditions of the nanowires. Comparison of the transients for GaAs nanowires (sample [nw1-C]) with those overcoated with an AlGaAs shell (sample [nw2-CS]) reveals an increase in overall carrier 


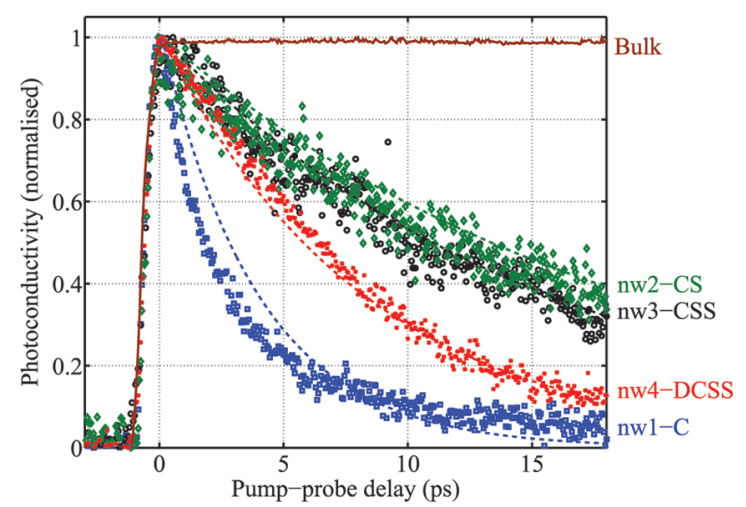

Figure 3. Normalized photoconductivity of four types of nanowires and bulk GaAs as a function of time after photoexcitation. The data from the different samples are distinguished by the following labels and colors: Bulk GaAs (Bulk, brown), core-shell NWs ([nw2-CS], green), core-shell-skin NWs([nw3-CSS], black), core-shell-skin NWs with high density of twin defects ([nw4DCSS], red), and core only NWs ([nw1-C], blue). All samples were excited at a wavelength of $810 \mathrm{~nm}$ with a fluence of $60 \mu \mathrm{J} / \mathrm{cm}^{2} /$ pulse corresponding to an areal photon density of around $2.4 \times$ $10^{14} \mathrm{~cm}^{-2}$. The risetime is likely to be resolution-limited, as the intrinsic onset of photoconductivity in bulk SI-GaAs is $\sim 100 \mathrm{fs}^{9}{ }^{9}$ The dashed lines are monoexponential fits as described in the text.

lifetime by approximately a factor of 4 through the overcoating technique. Similarly, changing from the one-temperature (sample [nw4-DCSS]) to the two-temperature (sample [nw3-CSS]) growth procedure clearly enhances the carrier lifetime by a significant amount. For a more quantitative characterization, a monoexponential of the form $\Delta E(\tau) / E$ $=A \exp \left(-\tau / \tau_{\mathrm{c}}\right)$ was fitted to the transients (indicated as dashed lines in Figure 3). The extracted photoconductivity lifetimes $\left(\tau_{\mathrm{c}}\right)$ are shown in Figure $4 \mathrm{a}$ for a range of incident fluences. It can be seen that the carrier lifetime increases with excitation fluence for all nanowire samples, suggesting the existence of trap states that begin to saturate toward higher photoinjected carrier density. ${ }^{11}$

In order to assess the effects of both overcoating and defect-free growth, we constructed a model that accounts for both trapping of charges and other, nonsaturable, freecharge annihilation routes. While the process of overcoating will have a significant impact on the density of available surface states, a change in growth conditions for the GaAs core will mainly affect the nonsaturable charge recombination pathways. Appropriate fitting of such a model to the data will therefore allow clear separation of the effects of growthtemperature variation and overcoating on charge lifetimes. Our calculations are based on the following coupled rate equations, which determine the density of mobile charge carriers, $N$, and the density of unoccupied trap states, $T$, as a function of time after excitation

$$
\begin{array}{rlrl}
\frac{\mathrm{d} N}{\mathrm{~d} t} & =-\frac{N}{\tau_{\text {intrinsic }}}-\frac{N}{\tau_{\mathrm{NW}}}-\gamma N T & N(0) & =N_{\mathrm{i}} \\
\frac{\mathrm{d} T}{\mathrm{~d} t} & =-\gamma N T & T(0) & =T_{\mathrm{i}}
\end{array}
$$

Here, the initially generated density of free charges, $N_{\mathrm{i}}$ is related to the absorbed photon density, $N_{\mathrm{abs}}=\chi N_{\mathrm{i}}$ through
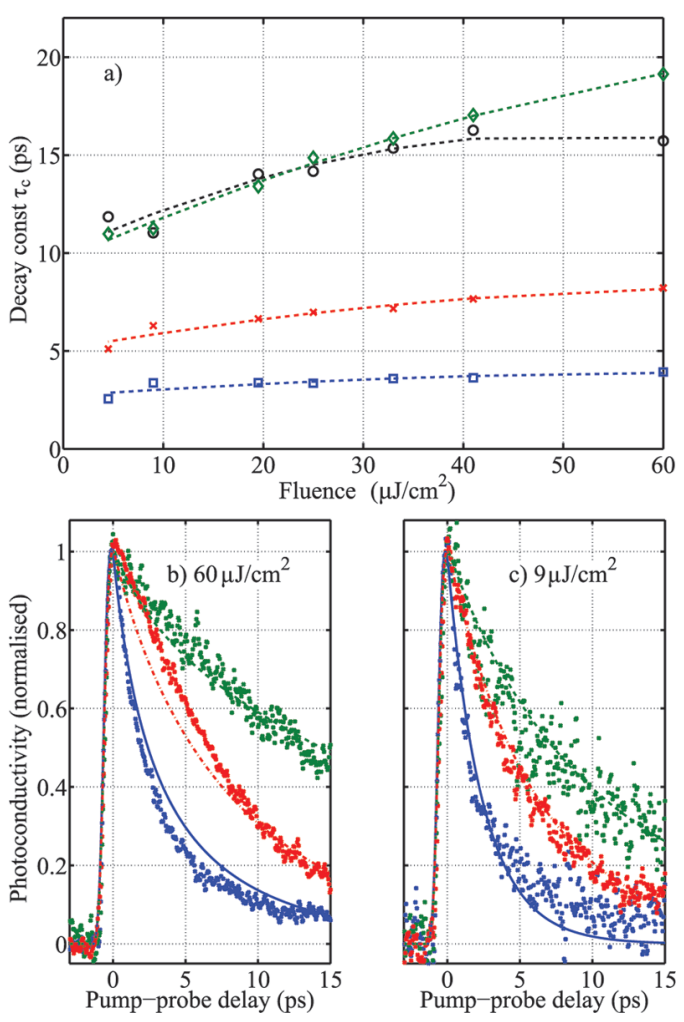

Figure 4. (a) Photoconductivity lifetime $\tau_{\mathrm{c}}$ is shown as a function of incident fluence for all four nanowire types; [nw1-C] ( $\square$ ), [nw2CS] $(\diamond)$, [nw3-CSS] $(\bigcirc)$ and [nw4-DCSS] $(\times)$. The dashed lines are a guide to the eye. (Below) Normalized photoconductivity transients measured at (b) high fluence $\left(60 \mu \mathrm{J} / \mathrm{cm}^{2} /\right.$ pulse) and (c) low fluence $\left(9 \mu \mathrm{J} / \mathrm{cm}^{2} /\right.$ pulse) along with the output of the numerical model (as described in the text) for three of the nanowire samples. The points represent the data while solid, dashed, and dot-dash lines are used for the numerical model for [nw1-C], [nw2-CS], and [nw4DCSS] nanowires, respectively.

a constant scaling factor $\chi$. The first term in eq 1 describes all bulk recombination routes through a time constant $\tau_{\text {intrinsic }}$ $=3.5 \mathrm{~ns}$, which was extracted from the photoconductivity decay of the bulk-GaAs sample (see Figure 3). The second term (incorporating $\tau_{\mathrm{NW}}$ ) accounts for additional nonsaturable carrier recombination routes that arise only in the nanowires. The third term describes charge trapping and recombination at available surface traps with a coupling constant given by $\gamma$. Such trapping reduces the free trap density $T$ by an equal amount, as indicated in eq 1 , with the initial trap density $T_{\mathrm{i}}$ being equal to the total density of traps (occupied or unoccupied) in the system. Solution of the coupled equations allowed the calculation of the time-dependent carrier density, which was convolved with a Gaussian (width $\sim 600 \mathrm{fs}$ ) to represent the response time of the experiment, and fitted to the photoconductivity transients. From such fits, values were obtained for all free parameters, that is, the nanowire-specific recombination time $\left(\tau_{\mathrm{NW}}\right)$, the total effective trap density $\left(\chi T_{\mathrm{i}}\right)$ and the coupling constant $(\gamma)$ for the trapping process.

The contributions arising from overcoating and changes in growth condition were separated by assuming that the nanowire-specific charge recombination time changes from $\tau_{\mathrm{NW}[1 T]}$ to $\tau_{\mathrm{NW}[2 \mathrm{~T}]}$ when moving from one- to two-temparature growth of the core, and that the initial trap density changes 
Table 1. Parameter Values Extracted by Fitting the Photoconductivity Decay Traces Shown in Figure 4b,c to the solutions of Equation 1

\begin{tabular}{ll}
\hline parameter & \multicolumn{1}{c}{ value } \\
\hline$\gamma$ & $1.62 \times 10^{-7} \mathrm{~cm}^{3} \mathrm{~s}^{-1}$ \\
$\tau_{\mathrm{NW}[1 \mathrm{~T}]}$ & $10.2 \mathrm{ps}$ \\
$\tau_{\mathrm{NW}[2 \mathrm{~T}]}$ & $28.2 \mathrm{ps}$ \\
$T_{\mathrm{i}[\mathrm{CS}]} / T_{\mathrm{i}[\mathrm{C}]}$ & 0.182
\end{tabular}

from $T_{\mathrm{i}[\mathrm{C}]}$ to $T_{\mathrm{i}[\mathrm{CS}]}$ when moving from core-only to overcoated nanowires. The coupling parameter $\gamma$ describing the interaction cross-section of charges with the traps was held constant between the fits. The model solutions were fit simultaneously to the photoconductivity traces for samples [nw1-C], [nw2CS], and [nw4-DCSS] at all excitation fluences investigated. Figure 4b,c shows the global fits to the data for two different excitation fluences with the extracted parameter values given in Table 1. The excellent agreement between the conductivity data and the model solutions validates our explanation that a reduction of core surface trap states occurs as a result of overcoating, and an increase in carrier lifetime arises from a reduction of twin-defects inside the core material.

Charge carriers in nanometer-sized objects are inherently more susceptible to interactions with surface states than those in bulk materials. ${ }^{3,12}$ Control of the surface chemistry is therefore essential in order for these objects to be implemented successfully in nanoscale devices. Chemical surface passivation of GaAs surfaces has previously been shown to reduce both the surface trap density ${ }^{13}$ and potentially also the surface recombination velocity. ${ }^{14}$ The latter has been attributed to a change in surface potential, and a subsequent reduction in the thickness of the surface depletion region. However, even with an optimized surface passivation protocol, the exciton-lifetime was not found to approach bulk standards. ${ }^{14}$ More recently, several groups have investigated the effect of overcoating nanowires with high-bandgap lattice-matched shells as an alternative to surface passivation. ${ }^{15,16}$ Using this technique, the luminescence emission efficiency was found to increase by 3 orders of magnitude (at cryogenic temperatures) and specific nanowires were demonstrated to have an emission lifetime approaching that of bulk GaAs. ${ }^{2}$ However, for use of such nanowires in electronics, the carrier mobility and lifetime at room temperature are the most important quantities to investigate. Our measurements and analysis demonstrate that at room temperature overcoating GaAs nanowires with a high-bandgap shell leads to an $82 \%$ reduction in the density of surface trap states. In addition, we find that changing from a onetemperature to a two-temperature growth procedure increases the nanowire-specific carrier recombination lifetime $\tau_{\mathrm{NW}}$ by $176 \%$, that is, from 10.2 to 28.2 ps.

In addition to the assessment of carrier lifetimes, we are able to determine the influence of growth techniques on the charge-carrier mobility in the nanowires. For this purpose, a second experimental approach was used in which the frequency-dependent conductivity is measured at a fixed delay after excitation. Figure 5 shows such data, collected at a delay of $2 \mathrm{ps}$ after photoexcitation in order to avoid the complications associated with temporal overlap of the pump

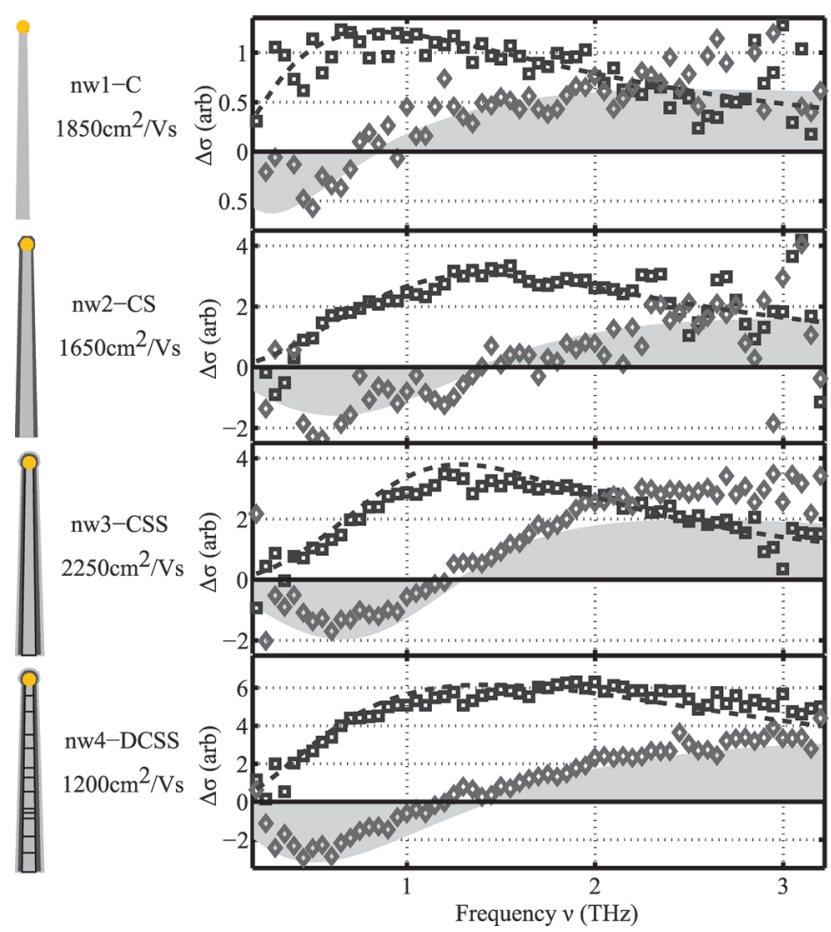

Figure 5. Terahertz-frequency photoconductivity spectra for all four types of nanowires. The data were taken at $\sim 2$ ps after excitation with at an excitation fluence of $227 \mu \mathrm{J} / \mathrm{cm}^{2} /$ pulse. The squares represent the real part of the conductivity data, while the diamonds represent the imaginary component of the data. The dashed lines and filled areas are fits given by the model presented in the text, which yield the charge-carrier mobilities displayed on the left.

and probe within the sample. ${ }^{17}$ A significant surface plasmon resonance $^{18}$ is seen between 1 and $2 \mathrm{THz}$ for all four types of nanowires in agreement with our previous measurements on GaAs nanowires. ${ }^{3}$ We are able to fit the experimental data assuming that the photoinduced conductivity $\Delta \sigma$ comprises both a Drude-like free-carrier response and a surface plasmon, ${ }^{3}$ that is, $\Delta \sigma=\left(\sigma_{\text {Drude }}+\sigma_{\text {Plasmon }}\right)$, where

$$
\begin{aligned}
\sigma_{\text {Drude }} & =\frac{\mathrm{i} N_{\mathrm{d}} e^{2} \omega}{m^{*}\left(\omega^{2}+\mathrm{i} \omega \Gamma\right)} \\
\sigma_{\text {Plasmon }} & =\frac{\mathrm{i} N_{\mathrm{p}} e^{2} \omega}{m^{*}\left(\omega^{2}-\omega_{0}^{2}+\mathrm{i} \omega \Gamma\right)}
\end{aligned}
$$

In these equations, $N_{\mathrm{d}}$ and $N_{\mathrm{p}}$ represent the carrier densities in the Drude mode and the plasmon mode respectively, $\Gamma$ is the momentum scattering rate and $\omega_{0}$ is the plasmon frequency. The center frequency of the surface plasmon is related to the carrier density by $\omega_{0}=\left(f N_{\mathrm{p}} e^{2} / m^{*} \varepsilon_{\mathrm{r}}\right)^{1 / 2}$, where $f$ is a geometrical factor $(1 / 2 \text { in the case of a cylinder })^{19}$ and $\varepsilon_{\mathrm{r}}$ is the terahertz-frequency electric permittivity of the material. Fitting these equations to the terahertz spectra (as shown in Figure 5) allows the momentum scattering rate to be extracted, from which the carrier mobility can be determined through

$$
\mu=\frac{e}{m^{*} \Gamma}
$$


The charge mobility values obtained in this manner are displayed in Figure 5 next to the respective photoconductivity spectra for all samples.

Changing the growth conditions from a one-temperature to a two-temperature procedure clearly has the most striking effect on charge mobility. Sample [nw4-DCSS] grown by a one-temperature procedure shows a mobility of $1200 \mathrm{~cm}^{2} /$ (V s) while reduction of twin-defect density through twotemperature growth (sample [nw3-CSS]) yields a mobility $2250( \pm 70) \mathrm{cm}^{2} /(\mathrm{V} \mathrm{s})$. This represents almost a doubling in room-temperature carrier mobility resulting from the improved growth techniques. Increases in carrier mobility through nearly defect-free growth have previously been postulated for GaN nanowires of over $100 \mathrm{~nm}$ diameter. ${ }^{20}$ Here we demonstrate that elimination of twin-defects results in a nearly 2-fold increase in mobility, or a halving of the momentum scattering rate, suggesting that momentum scattering processes from twin-defects can have a significant impact on material performance.

The effects of overcoating of the nanowires on the chargemobility are more subtle. While overcoating of the GaAs core with AlGaAs appears to cause a slight drop in mobility from $\sim 1850$ to $\sim 1650 \mathrm{~cm}^{2} /(\mathrm{V} \mathrm{s})$, additional overcoating with a GaAs skin leads to an increase to $2250 \mathrm{~cm}^{2} /(\mathrm{V} \mathrm{s})$. The slight decrease in mobility upon overcoating the GaAs core with AlGaAs may be caused by the adsorption of oxygen into the AlGaAs shell. Adsorped oxygen may act both as a scattering site for carriers and an exciton recombination site. ${ }^{2}$ The subsequent increase in mobility following additional overcoating with GaAs may prevent such oxidation and also lead to a change in surface field, the thickness of the depletion region, or the nature of momentum scattering from the interface. Further investigations will be required to elucidate the microscopic origin of the changes in mobility with overcoating.

In conclusion, we have demonstrated that both twotemperature growth techniques and overcoating with a higher bandgap semiconductor significantly enhance the performance of GaAs nanowires. Our contactless terahertz conductivity measurements show that reduction of twin-defect states through two-temperature growth of the GaAs core leads to an almost doubling of the charge-carrier mobility and a tripling of the (nonsaturable) carrier lifetime. Surface states were found to have a strong impact on the electronic properties of these nanosized objects, causing a nonlinear, saturable component in the charge dynamics. However, overcoating the GaAs core reduces the density of such surface traps by $82 \%$, resulting in longer-lived charge conductivity. Both techniques represent a significant step toward achieving bulklike material quality for nanomaterials, and point toward successful application of these nanowires in a wide variety of high-mobility, high speed optoelectronic devices.

Acknowledgment. The authors thank the EPSRC (UK) (P.P., L.M.H., M.B.J.), the Australian Research Council, and the Australian National Fabrication Facility (H.J., Q.G., H.H.T., C.J.) for funding.

Supporting Information Available: Details of the opticalpump terahertz-probe experimental apparatus. This material is available free of charge via the Internet at http:// pubs.acs.org.

\section{References}

(1) Li, Y.; Qian, F.; Xiang, J.; Lieber, C. M. Mater. Today 2006, 9, 18.

(2) Perera, S.; Fickenscher, M. A.; Jackson, H. E.; Smith, L. M.; Yarrisonrice, J. M.; Joyce, H. J.; Gao, Q.; Tan, H. H.; Jagadish, C.; Zhang, X.; Zou, J. Appl. Phys. Lett. 2008, 93, 053110.

(3) Parkinson, P.; Lloyd-Hughes, J.; Gao, Q.; Tan, H. H.; Jagadish, C.; Johnston, M. B.; Herz, L. M. Nano Lett. 2007, 7, 2162-2165.

(4) Joyce, H. J.; Gao, Q.; Tan, H. H.; Jagadish, C.; Kim, Y.; Zhang, X.; Guo, Y. N.; Zou, J. Nano Lett. 2007, 7, 921-926.

(5) Paladugu, M.; Zou, J.; Guo, Y. N.; Zhang, X.; Joyce, H. J.; Gao, Q.; Tan, H. H.; Jagadish, C.; Kim, Y. Angew. Chem., Int. Ed. 2009, 48, $780-783$.

(6) Joyce, H. J.; Gao, Q.; Tan, H. H.; Jagadish, C.; Kim, Y.; Fickenscher, M. A.; Perera, S.; Hoang, T. B.; Smith, L. M.; Jackson, H. E.; Yarrisonrice, J. M.; Zhang, X.; Zou, J. Adv. Funct. Mater. 2008, 18, 37943800.

(7) Tambe, M. J.; Lim, S. K.; Smith, M. J.; Allard, L. F.; Gradecak, S. Appl. Phys. Lett. 2008, 93, 151917.

(8) Kim, Y.; Joyce, H. J.; Gao, O.; Tan, H. H.; Jagadish, C.; Paladugu, M.; Zou, J.; Suvorova, A. A. Nano Lett. 2006, 6, 599.

(9) Huber, R.; Tauser, F.; Brodschelm, A.; Bichler, M.; Abstreiter, G.; Leitenstorfer, A. Nature 2001, 414, 286-289.

(10) Kužel, P.; Kadlec, F.; Němec, H. J. Chem. Phys. 2007, 127, 11.

(11) Jepsen, P. U.; Schairer, W.; Libon, I. H.; Lemmer, U.; Hecker, N. E.; Birkholz, M.; Lips, K.; Schall, M. Appl. Phys. Lett. 2001, 79, 1291.

(12) Cooke, D. G.; MacDonald, A. N.; Hryciw, A.; Meldrum, A.; Wang, J.; Li, Q.; Hegmann, F. A. J. Mater. Sci.: Mater. Electron. 2007, 18, S447-S452.

(13) Lloyd-Hughes, J.; Merchant, S. K. E.; Fu, L.; Tan, H. H.; Jagadish, C.; Castro-Camus, E.; Johnston, M. B. Appl. Phys. Lett. 2006, 89, 232102.

(14) Ogawa, K.; Haraguchi, K.; Hiruma, K.; Fujisaki, Y.; Katsuyama, T.; Fasol, G. J. Lumin. 1992, 53, 387-390.

(15) Sköld, N.; Karlsson, L. S.; Larsson, M. W.; Pistol, M. E.; Seifert, W.; Trägårdh; Samuelson, L. Nano Lett. 2005, 5, 1943-1947.

(16) Noborisaka, J.; Motohisa, J.; Hara, S.; Fukui, T. Appl. Phys. Lett. 2005, 87, 093109.

(17) Nienhuys, H. K.; Sundström, V. Phys. Rev. B 2005, 71, 235110.

(18) Nienhuys, H. K.; Sundström, V. Appl. Phys. Lett. 2005, 87, 012101.

(19) Pitarke, J. M.; Silkin, V. M.; Chulkov, E. V.; Echenique, P. M. Rep. Prog. Phys. 2007, 70, 1-87.

(20) Motayed, A.; Vaudin, M.; Davydov, A. V.; Melngailis, J.; He, M. Q.; Mohammad, S. N. Appl. Phys. Lett. 2007, 90, 043104.

NL9016336 